\title{
Klebrige Nascherei für Rüsselspringer: Elephantulus edwardii als Bestäuber von Whiteheadia bifolia
}

\author{
Petra Wester
}

\begin{abstract}
Following the recent discovery of rodent pollination in the Pagoda lily (Whiteheadia bifolia, Hyacinthaceae) in South Africa, now the Cape rock elephant-shrew (Elephantulus edwardii, order Macroscelidea) is reported as an additional pollinator. The animals, live-trapped near W. bifolia plants, were released in two terraria, containing the plants. The elephant-shrews licked nectar with their long and slender tongues while being dusted with pollen and touching the stigmas of the flowers with their long and flexible noses. The captured elephant-shrews had W. bifolia pollen in their faeces, likely as a result of grooming their fur as they visited the flowers without eating or destroying them. This is the first record of pollination and nectar-consumption in the primarily insectivorous E. edwardii.
\end{abstract}

\section{Zusammenfassung}

Nachdem Whiteheadia bifolia (Pagoden-Lilie, Hyacinthaceae) vor kurzem in Südafrika als nagerbestäubt identifiziert wurde, wird jetzt der Kap-Rüsselspringer (Elephantulus edwardii, Ordnung Macroscelidea) als weiterer Bestäuber beschrieben. Nahe Whiteheadia-Pflanzen gefangene Tiere wurden in zwei Terrarien gegeben, welche die Pflanzen enthielten. Während die Rüsselspringer Nektar mit ihren langen dünnen Zungen aufleckten, wurden sie mit Pollen eingestäubt und berührten die Narben der Blüten mit ihren langen und beweglichen Nasen. Die gefangenen Tiere hatten W. bifolia-Pollenkörner in ihrem Kot, vermutlich als Resultat des Fellputzens, da sie die Blüten besuchten, ohne sie zu fressen oder zu zerstören. Dies ist die erste Beobachtung von Bestäubung und Nektarkonsum beim vorwiegend insektivoren Kap-Rüsselspringer.

\section{Whiteheadia bifolia, Mäuse und Rüsselspringer}

Die Pagoden-Lilie (Whiteheadia bifolia) ist auf die aride Winterregen-Region Südafrikas und Süd-Namibias beschränkt und wächst an geschützten schattigen Felsstandorten. Der ca. $20 \mathrm{~cm}$ hohe Zwiebelgeophyt sieht sehr bizarr aus mit seinen beiden fleischigen, flach auf dem Boden liegenden Blättern und der zentralen vielblütigen Infloreszenz mit langen grünen Traglättern. Die unauffälligen grünlichen Blüten bilden extrem viskosen, klebrigen Nektar. Vor kurzem wurde entdeckt, dass die Art von nachtaktiven Nagern bestäubt wird (WESTER 2009 a, b). Nagerbestäubung ist ein seltenes Phänomen und nur von wenigen Pflanzenarten in Südafrika und im tropischen Afrika, China, Indien, Malaysia, Australien und den Neotropen bekannt (WeSTER 2009 b).

In der oben genannten Whiteheadia-Studie wurden Namaqua-Felsenmäuse (Aethomys namaquensis, Muridae) im Gelände und im Labor beobachtet. Die Mäuse bestäubten die Blüten dieser Art, während andere Tiere (Insekten, Vögel) nie an den Blüten beobachtet wurden. Whiteheadia bifolia hat ähnliche Blütenmerkmale wie andere Pflanzenarten mit so genanntem Nagerbestäubungs-Syndrom: Bodennahe, visuell unauffällige, robuste, napfförmige Blüten mit leicht zugänglichem Nektar, exponierte Staubblätter und Griffel sowie auffälliger Duft.

Anschließendes Fangen im darauffolgenden Jahr lieferte (neben weiteren Namaqua-Felsenmäusen) zwei Individuen des Kap-Rüsselspringers (Elephantulus edwardii), ein endemischer Kleinsäuger Südafrikas. Der englische Trivialname „elephant shrew“ (wortgetreu übersetzt Elefanten-Spitzmaus) deutet auf die Ähnlichkeit zwischen ihren langen, dünnen, beweglichen Nasen und dem Rüssel eines Elefanten hin. Aufgrund oberflächlicher Ähnlichkeit wurde eine Verwandtschaft mit Spitzmäusen vermutet. Da sie jedoch mit letzteren nicht näher verwandt sind, benutzen viele Autoren das Bantu-Wort Sengi. Die Rüsselspringer oder Sengis gehören zur Ordnung der Macroscelidea (Familie Macroscelididae) und umfassen derzeit 17 Arten in vier Gattungen,

Abb. 1 (Seite 123): Mit seiner langen, rosafarbenen Zunge leckt der Kap-Rüsselspringer Nektar aus der W. bifolia-Blüte. Man sieht den Pollen auf der langen Nase. 
die in Afrika verbreitet sind (RAThBUn 2009). Vorwiegend auf morphologischen Merkmalen basierend wurde angenommen, dass die Rüsselspringer zu den Hasenartigen und Nagern (inklusive Mäusen) gehören. Daten von Fossilien und molekularen Untersuchungen legen aber nahe, dass die Sengis zu den Afrotheria (inklusive Erdferkel, Tenreks, Goldmulle, Schliefer, Seekühe und Elefanten) gehören. Die langen Nasen der Tiere erinnern jedoch auch an die berüchtigten, fiktiven Naslinge (Ordnung Rhinogradentia nach STÜMPKE 1961).

Da Rüsselspringer den Mäusen in Gestalt und Größe ähnlich sind, könnte man sich vorstellen, dass auch sie als Bestäuber in Frage kommen, jedoch wären sie aufgrund ihrer vorwiegend insectivoren Ernährungsweise eher unerwartete Blütenbesucher. $\mathrm{Da}$ so gut wie nichts über ihr Verhalten an Blüten bekannt ist, wurden Untersuchungen durchgeführt, die klären sollten, ob sie ebenfalls Bestäuber von W. bifolia sind.

\section{Beobachtung von Rüsselspringern im Terrarium}

In den Cederbergen Südafrikas (am Sevilla rock art-Wanderweg nahe Clanwilliam) wurden, wie schon in der oben genannten Studie, auch im Jahr 2009 etwa 40 mit Erdnussbutter versehene Lebendfallen drei Tage und Nächte lang aufgestellt. Neben 22 Namaqua-Felsenmäusen wurden diesmal auch zwei Kap-Rüsselspringer gefangen. Die Rüsselspringer wurden in zwei Glasterrarien gesetzt. Jedes der Terrarien war mit Sand, Steinen und W. bifolia-Pflanzen bestückt. Über vier Tage hinweg wurden die Tiere zwischen Mittag und Mitternacht insgesamt 14 Stunden lang beobachtet. Die Rüsselspringer versteckten sich zunächst zwischen den Steinen. Nach etwa einer halben Stunde kamen sie hervor und besuchten die Blüten. Mehr als 50 Blütenbesuche konnten zwischen 14 und 23 Uhr an vier Tagen beobachtet werden. Die Tiere liefen nacheinander zu den verschiedenen Blütenständen und besuchten jeweils einige Blüten pro Blütenstand. Sie steckten ihre langen und flexiblen $\mathrm{Na}$ -

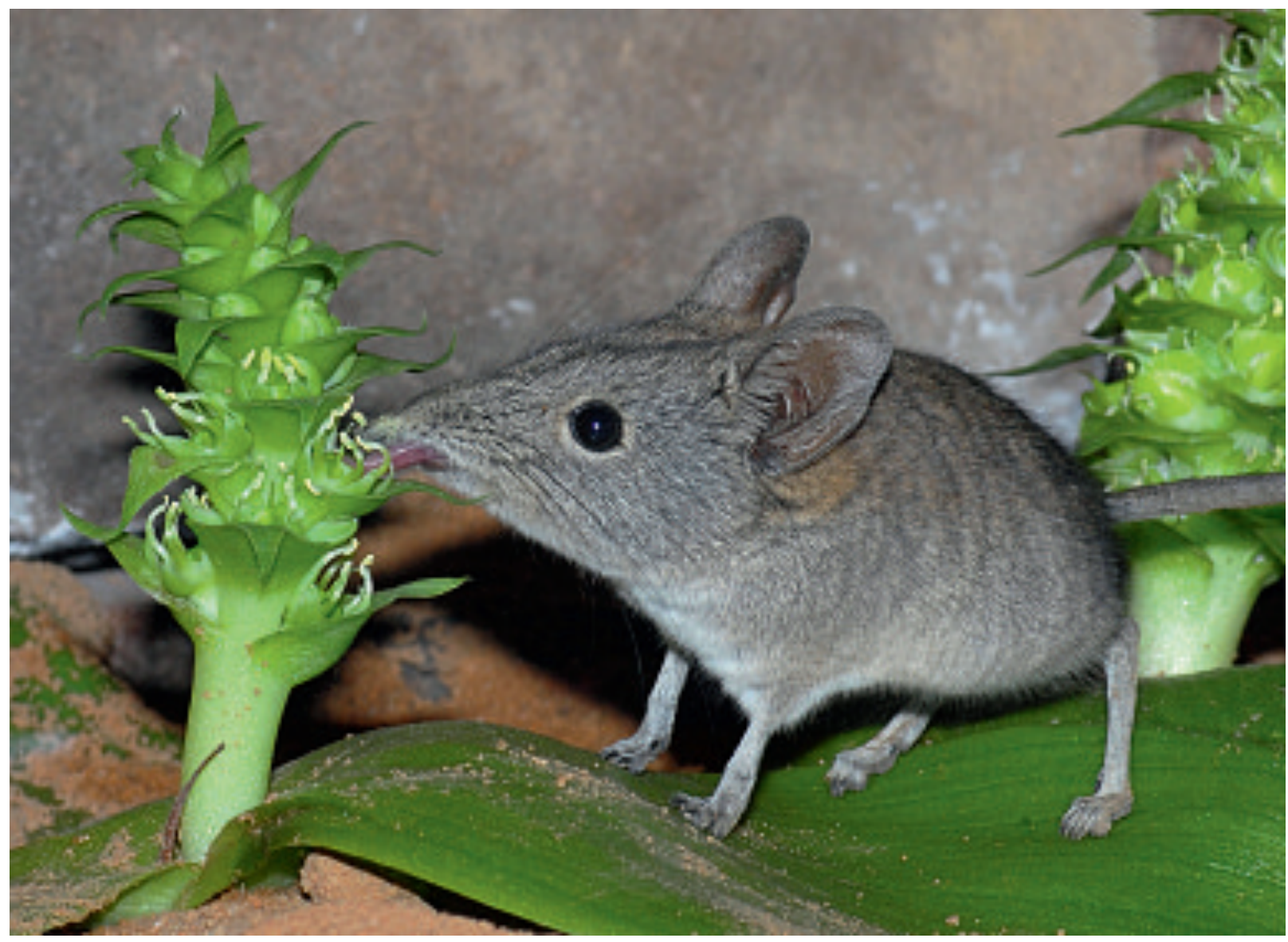




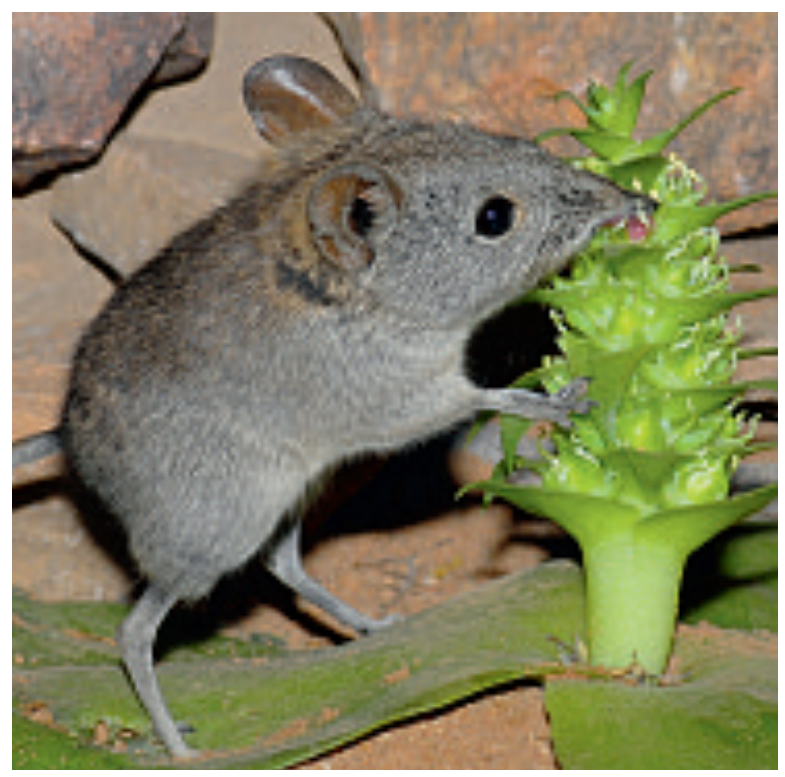

sen zwischen die Staubblätter und leckten den viskosen Nektar zwischen Fruchtknoten und Staubblättern mit ihren sehr langen und schmalen Zungen. Dabei berührten sie stets die Pollensäcke und wurden sichtbar mit Pollen auf der langen Nase eingestäubt. Sie kamen auch mit den Narben der Blüten in Kontakt. Die Rüsselspringer lecken den Nektar, ohne dabei aktiv Pollen zu fressen oder die Blüten zu beschädigen oder zu fressen. Manchmal putzten die Tiere zwischen den Blütenbesuchen ihr Fell.

\section{Pollenkörner im Kot der Rüsselspringer}

Der aus den Fallen entnommene Kot der Rüsselspringer wurde zerkleinert, mit Fuchsin (färbt Pollen rot) versehen und mikroskopiert. Die beiden Kotproben enthielten sehr große Mengen an W. bifolia-Pollen und nur sehr geringe Mengen von anderen Pflanzen. Letzteres ist erklärbar mit der Tatsache, dass der KapRüsselspringer ein Allesfresser ist, also neben Insekten auch Pflanzenmaterial frisst. Die W. bifolia-Pollenkörner im Kot sind jedoch eher das Resultat von Putzbewegungen.

\section{Rüsselspringer - kaum erforschte Bestäuber}

Daten zu Rüsselspringern als Bestäuber sind extrem rar. Blütenbesuche wurden indirekt von Wiens et al. (1983) nachgewiesen. Sie fanden Protea-Pollen auf den Nasen und im Kot von nahe nagerbestäubten Protea-Pflanzen gefangenen Kap-Rüsselspringern. In dieser Studie leckten die gefangenen Tiere jedoch nur selten an den Blütenständen, aber nie Nektar vom Nektarreservoir der Blüten, die ihnen präsentiert wurden. Fleming \& Nicolson (2002) fanden ebenfalls Pollen der nagerbestäubten Protea humiflora auf den Nasen und im Kot von KapRüsselspringern. Sie schlossen aber daraus, dass sich die Tiere von Insekten ernährten, welche sich in den Blütenständen befanden. Diese Schlussfolgerung basiert vermutlich auf der Tatsache, dass man zu dieser Zeit Rüsselspringer für streng insektivor hielt (PERRIN 1997).

Die vorliegende Studie ist die erste, die eindeutig zeigt, dass der Kap-Rüsselspringer Blüten besucht, um Nektar zu trinken. Von einer verwandten Art, dem Kurznasen- Rüsselspringer (E. brachyrhynchus) wurde vor kurzem gezeigt, dass sie Blüten des Parasiten Cytinus visseri (Zistrosenwürger) besucht und wahrscheinlich bestäubt (JoHnson et al. 2011). Es ist nicht auszuschließen, dass weitere Rüsselspringer-Arten Blüten bestäuben.

Wie auch Nager sind Rüsselspringer eine weitere Gruppe von bestäubenden Tieren, die man als „nichtfliegende Säuger“ bezeichnet, um sie von Fledermäusen abzugrenzen. Diese Gruppe umfasst auch Beuteltiere und Affen (CARTHEw \& Goldingay 1997). Die Pflanzen haben einige Blütenmerkmale gemeinsam, die auf Anpassung an diese Tiere schließen lassen und die das Bestäubungssyndrom Therophilie bilden (Rebelo \& BreytenbaCH 1987). Allerdings variiert die Morphologie und das Verhalten dieser Säuger gewaltig, was in einer großen Spannweite von Blüten-Vielfalt innerhalb des Syndroms zum Ausdruck kommt. Zum Beispiel sind Blüten, die von Affen bestäubt werden, normalerweise sehr groß und Beuteltier-Blüten sind normalerweise im Kronendach zu finden. Demgegenüber scheinen nagerbestäubte Blüten eher klein und in Bodennähe zu sein (Turner 1982, Kress et al. 1994, Wester a, b. 2009). Rüsselspringer sind den Nagern in Größe und Blütenbesuchs-Verhalten ähnlich. Es wird vermutet, dass es keine auf Rüsselspringer spezialisierte Pflanzen gibt, sondern dass sie sich diese mit Mäusen als Bestäuber teilen. 


\section{Literatur}

Carthew, S. M. \& Goldingay, R. L. 1997: Non-flying mammals as pollinators. - TREE 12: 104-108.

Fleming, P. A. \& Nicolson, S. W. 2002: How important is the relationship between Protea humiflora (Proteaceae) and its non-flying mammal pollinators? - Oecologia 132: 361-368.

Johnson, S. D., Burgoyne, P. M., Harder, L. D. \& Dötterl, S. 20I I: Mammal pollinators lured by the scent of a parasitic plant. - Proc. R. Soc. Lond. 278: 2303-2310. Kress, W. J., Schatz, G. E., Andrianifahanana, M., Morland, H .S. I994: Pollination of Ravenala madagascariensis (Strelitziaceae) by lemurs in Madagascar: evidence for an archaic coevolutionary system. - Amer. J. Bot. 81: 542-551.

Perrin, M. 1997. Cape rock elephant shrew, Elephantulus edwardii. - In: Mills G. \& Hes, L. (Hrsg.): The complete book of southern African mammals. - Kapstadt.

Rathbun, G. B. 2009: Why is there discordant diversity in sengi (Mammalia: Afrotheria: Macroscelidea) taxonomy and ecology? - Afr. J. Ecol. 47: 1-13.

Rebelo, A. G. \& Breytenbach, G. J. 1987: Mammal pollination in the Cape flora. In: Rebelo, A.G. (Hrsg.): A preliminary synthesis of pollination biology in the Cape flora. South Afric. Nat. Sci. Program Report 141: 109-123. Turner, V. I982: Marsupials as pollinators in Australia. In: Powell, J. A. \& Richards, R. J. (Hrsg.): Pollination and evolution. - Sydney.

Wester, P. 2009 a: Mice pollinators in the Cederberg. The first field observations with photographic documentation of rodent pollination in South Africa. - Veld \& Flora 95:

82-85.

Wester, P. 2009 b: Von Mäusen und Pavianschuhen. Erste Geländebeobachtung von Nager-Bestäubung in Südafrika. - Palmengarten 73: 120-125.

Wiens, D., Rourke, J., Casper, B., Rickart, E., Lapine, T., Peterson, C. \& Channing, A. 1983: Nonflying mammal pollination of southern african proteas: a non-coevolved system. - Ann. Missouri Bot. Gard. 70: 1-31.

Abb. 2 (Seite 124): Der Kap-Rüsselspringer lehnt nektarleckend am Blütenstand von W. bifolia. Seine Nase berührt die Pollensäcke der Blüten.

Abb. 3 (oben): Der Kap-Rüsselspringer berührt die Pollensäcke und wird auf der Nase mit Pollen eingestäubt.

Abb. 4 (Mitte): Ein gähnender Kap-Rüsselspringer, seine Nase nach oben biegend und seine lange, dünne Zunge zeigend (hier nicht völlig ausgestreckt).

Abb. 5 (unten): Whiteheadia in einer Felsspalte.
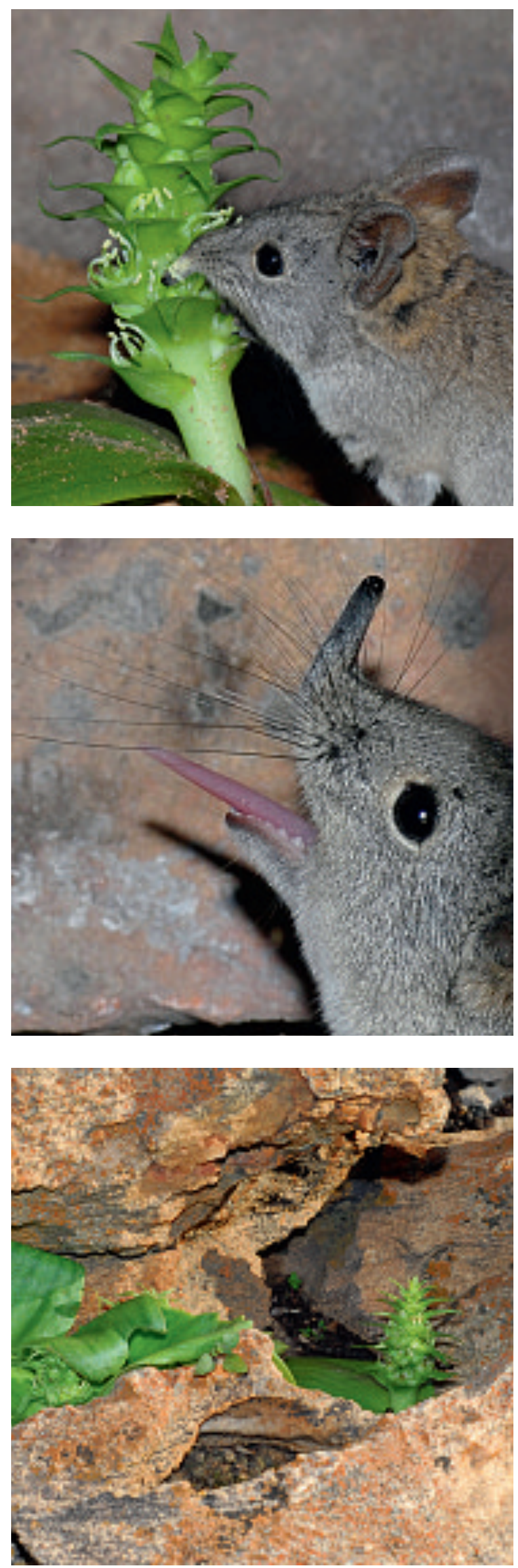